\title{
Universidad y Violencia de Género: Experiencia en Estudiantes Universitarios de Trabajo Social en la Región de Tarapacá
}

\section{University and Gender Violence: Experience in University Social Work Students in the Tarapacá Region}

\author{
Carolina Garcés Estrada* \\ Antonia Santos Pérez \\ Loreto Castillo Collado \\ Universidad Arturo Prat, Chile
}

\begin{abstract}
La violencia de género es un problema social que afecta mayoritariamente a las mujeres. Estudiar este fenómeno es relevante en el ámbito universitario para dar cuenta de su existencia y magnitud. En este artículo se exponen el análisis y los resultados de un estudio descriptivo, de tipo cuantitativo, cuyo objetivo fue evidenciar las diversas expresiones de violencia de género en una universidad pública en el norte de Chile en la carrera de Trabajo Social. Sobre la base del trabajo de campo realizado a partir de una encuesta dirigida a las y los estudiantes, los resultados muestran como la violencia de género está presente en los espacios y prácticas educativas, formando parte de un continuo de múltiples agresiones en diferentes contextos de socialización y particularmente encontrándose soterradas en las experiencias formativas de los y las estudiantes. Asimismo, se relevan las diferentes manifestaciones, escenarios y actores en las prácticas de violencia en la universidad. Como conclusión se plantea que las instituciones de educación superior son espacios educativos donde se deben establecer estrategias institucionales para actuar en favor de la educación en igualdad, la inclusión y la erradicación de cualquier forma de agresión que atente contra la libertad e igualdad de las personas.
\end{abstract}

Descriptores: Violencia; Educación; Universidad; Sexismo, Trabajo social.

Gender violence is a social problem that mainly affects women. Studying this phenomenon is relevant in the university environment to account for its existence and magnitude. This article presents the analysis and results of a descriptive, quantitative study, which aimed at showing the various expressions of gender violence in a public university in northern Chile in the Social Work program. On the basis of field work carried out from a survey directed to students, the results show how gender violence is present in educational spaces and practices, forming part of a continuum of multiple aggressions in different socialization contexts and particularly finding themselves buried in the formative experiences of the students. Likewise, the different manifestations, scenarios and actors in the practices of violence in the university are surveyed. As a conclusion, it is suggested that Higher Education institutions are educational spaces where institutional strategies must be established to act in favor of education in equality, inclusion and the eradication of any form of aggression that threatens the freedom and equality of people.

Keywords: Violence; Education; University; Sexism, Social work.

*Contacto: carogarcesestrada@gmail.com

ISSN: 0718-7378

www.rinace.net/rlei/
Recibido: 27/05/2020

$1^{\text {a }}$ Evaluación: 10/08/2020

Aceptado: 30/08/2020 


\section{Revisión de la literatura ${ }^{1}$}

La violencia contra las mujeres y población LGTBI no es ni un fenómeno nuevo ni una realidad que tienda a disminuir (Bosch-Fiol y Ferrer-Pérez, 2012). La preocupación reciente de las universidades respecto a la discriminación y violencia por razones de género ha llevado a la formulación de diferentes programas y políticas orientadas a atender esta situación y promover cambios al interior de las instituciones de educación superior (Buquet et al., 2014). No obstante, se observa un lento avance en el reconocimiento del sexismo y sus repercusiones, además de los escasos estudios realizados sobre expresiones de violencia referidos a campos de estudios universitarios. Las medidas implementadas deben comprender la violencia al interior de las universidades como un grave problema de derechos humanos, que requiere de respuestas urgentes con el fin de promover cambios en estos espacios de formación y en la convivencia social (Buquet, 2011). Su abordaje no ha sido suficientemente efectivo debido a la intervención de diferentes patrones culturales y marcos normativos que niegan o relativizan su importancia.

Esta investigación se realiza a partir de la instalación de las demandas estudiantiles frente a la necesidad de crear protocolos y sanciones explícitas a quienes incurran en conductas de discriminación, acoso y violencia en los espacios universitarios. Estas demandas abrieron un contexto propicio para indagar en las valoraciones, experiencias y formas en que este fenómeno afecta a quienes forman parte de las instituciones de educación superior. Este estudio es parte de una investigación más amplia en el análisis de la violencia de género en varias carreras universitarias.

La carrera de Trabajo Social es parte de este grupo estudiado, presentando características que hacen que sea una población objeto relevante de análisis. En este sentido, podemos destacar el hecho que es una disciplina históricamente feminizada y cuyo fundamento tiene desafíos éticos, teóricos e instrumentales sobre las condiciones de desigualdad y la fragmentación de la sociedad (Carballeda, 2017), que suelen estar ligados a la propia trayectoria de vida de los y las estudiantes, marcada por eventos críticos que afectan tanto la estructura de personalidad, como al aprendizaje transgeneracional de ciertas pautas de relación que se reproducen en la vida adulta. Por otra parte, esta experiencia vital ha desarrollado un marcado énfasis en la defensa de los derechos de las personas, lo que pone en tensión la articulación entre sus propias trayectorias con su proceso formativo. Por consiguiente, la formación del Trabajo Social debe tener en consideración estas estrategias en la configuración de su quehacer disciplinario, sobre todo, si se quiere contribuir a las transformaciones sociales (Vivero, 2017).

Este artículo comienza con una aproximación al concepto de violencia de género enmarcado en las tensiones que se generan en las relaciones sexo/género desde un sistema heteronormado donde se sitúa la violencia de género y el sexismo en la Educación Superior. Posteriormente, se presenta la metodología implementada para dar paso a la exposición de los resultados. Finalmente se presentan las conclusiones, con un énfasis en el reconocimiento del sexismo y la necesidad de erradicarlo de los espacios universitarios.

\footnotetext{
${ }^{1}$ Este estudio se enmarca en una investigación más amplia titulada "Estudio sobre Violencia en la Facultad de Ciencias Jurídicas y Políticas de la Universidad Arturo Prat”, con financiamiento de la propia Universidad.
} 


\subsection{El continuo de la violencia de género}

El cuestionamiento creciente de la conformación de las relaciones de género conlleva la deslegitimación del patriarcado y consecuentemente a la emergencia del conflicto social entre hombres y mujeres, donde la violencia de género resultante no es un hecho aislado ni particular (Castells y Subirats, 2010). Su comprensión requiere situarla dentro de un parámetro estructural (Troncoso, Follegati y Stutzin, 2019) que nos permita observar y cuestionar las bases de la conformación de las relaciones sociales. Todo sistema social se configura desde la definición de las interacciones de los sujetos que lo conforman. Las sociedades modernas se disponen desde la representación del sistema patriarcal, configurándose históricamente a partir de definir relaciones de poder desde la preeminencia de lo masculino y articuladas desde la heterosexualidad. Este sistema asentado sobre las relaciones entre hombres y mujeres se mantiene en base a un entramado de estructuras materiales y simbólicas que impregnan las relaciones políticas, económicas, sociales y culturales del conjunto de la sociedad. Así el patriarcado es clave para dar cuenta de cómo se conforma y distribuye socialmente de forma desigual el poder entre los sexos. Como resultante, la opresión de género (Arruza, 2017) surge como un fenómeno que no puede reducirse a las relaciones interpersonales, sino que tiene una consistencia de carácter social en el que se reproducen jerarquías sociales impregnando normas e instituciones sociales.

La violencia de género es un fenómeno social ligado a la dominación masculina, indispensable para la producción y reproducción del sistema social patriarcal, siendo un hecho político al evidenciarse que las mujeres son objeto de una violencia específica, con un significado determinado otorgado por un marco interpretativo concreto del patriarcado (Cobo, 2019). El hecho de ser mujer constituye un posicionamiento social diferenciado del hecho de ser hombre en todos los ámbitos de las relaciones e instituciones sociales, normadas desde la subordinación de lo femenino. Así la violencia se constituye como una herramienta fundamental en el orden social patriarcal donde la mujer es sometida en tanto que objeto de dominación, negándole la libertad de sujeto social. En este sentido, la violencia hay que entenderla desde su carácter instrumental (Arendt, 2018) al ejercerse a partir de patrones estereotipados como mensajes, valores, íconos o signos que transmiten y reproducen las relaciones sociales en base a la relación sexo/género. Al mismo tiempo, la violencia de género es expresiva (Segato, 2016) asentándose en el territorio de interlocución que conforman el corporativo normado por el mandato de masculinidad. Al ser un medio de control social ejerce un papel fundamental en la naturalización de los comportamientos masculinos violentos y en la sumisión de otras personas producto de dichos comportamientos, como las niñas, mujeres y otras sexualidades no hegemónicas ni heteronormadas, expresiones del sexismo.

Los procesos actuales de globalización redefinen también los lugares "naturales" de mujeres y hombres, produciendo varios fenómenos de (des)reestructuración, razón por la que los modos de la violencia antes naturalizados y normalizados se hacen más evidentes. Asimismo, se han propiciado cambios en el entramado social, institucional y simbólico que van generando nuevos espacios de violencia y conflicto social entre los géneros (De Miguel, 2015).

\subsection{La tensión en las relaciones sexo/género desde la heteronormatividad}

Desde el análisis de las interacciones de género Osborne (2009) muestra como la violencia contiene cuatro componentes; responde a un fenómeno estructural, es un mecanismo de 
control de todas las mujeres, representa un persistente extremo de las conductas consideradas como normales y se observa una gran tolerancia hacia la misma. Estos elementos se sitúan en los procesos de socialización basados en el eje sexo/género que propician la diferenciación de los atributos sociales femeninos y masculinos. Siguiendo estas ideas, Connell y Messerschmidt (2005) observan la masculinidad hegemónica como un modelo de socialización que describe el modo de "ser hombre", valorado socialmente y punto de referencia para los demás. Desde esta perspectiva, la noción de violencia de género trasciende la violencia ejercida contra las mujeres e incluye también a todos aquellos cuerpos que no cumplen con los estereotipos esperados de masculinidad. A la vez que legitima ideológicamente la subordinación de las mujeres en su multiplicidad, y de otros grupos que no se adecúan a la masculinidad hegemónica.

En este modelo se construyen las identidades y se dan las pautas conformadoras para el llamado proceso de masculinización, y al mismo tiempo de feminización (Castells y Subirats, 2010). De manera similar, Segato (2016) plantea que esta socialización diferencial de los géneros provoca una especie de pedagogía de la crueldad en la que los hombres deben tributarse los cuerpos de las mujeres como parte de su afirmación y de la necesaria actualización de su identidad, principalmente en las últimas décadas en las que las mujeres como género están practicando formas y modos de vida que no se corresponden con el esquema tradicional que les asigna el orden social patriarcal. Este orden jerárquico, se expresa cotidianamente en las diversas formas de discriminación y violencias de género, la llamada “dueñidad" (Segato, 2016) de los hombres sobre el cuerpo de las mujeres. El modelo hegemónico de la conducta sexual masculina es, además, androcéntrico y contiene en sí mismo una tensión entre el poder masculino y el poder femenino.

La violencia de género se expresa cotidianamente a través de comportamientos sexistas que discriminan, ignoran, someten y subordinan a las mujeres en los diferentes aspectos de su existencia. Son agresiones materiales y simbólicas que afectan la libertad, dignidad, seguridad, intimidad e integridad moral y/o física de las mujeres (Velázquez, 2006). En las instituciones educativas la violencia ha estado normalizada, velada y silenciada. Las distintas expresiones de la violencia irrumpen en todos los espacios de las relaciones sociales y las universidades no están inmunes a este fenómeno.

\subsection{Violencia de género y sexismo en la educación superior}

La significativa irrupción de la violencia como un problema social en contextos educativos guarda relación también con una mayor capacidad de detección y de sensibilización ante esta problemática. Los movimientos feministas en las universidades han denunciado la violencia (Troncoso, Follegati y Stutzin, 2019) haciendo visibles sus expresiones e impulsando un espíritu de transformación social que pone de manifiesto la necesidad de implementar medidas que apunten a su prevención y erradicación (Santos, 2019).

Distintos estudios (Mingo y Moreno, 2017) documentan el hostigamiento sexual, físico y verbal que enfrentan muchas mujeres en las aulas, pasillos y en los distintos espacios de las instituciones educativas. Estos comportamientos registran insultos, gritos, miradas insidiosas, chistes, gestos, burlas, "piropos", comentarios, rumores, críticas, chismes, insinuaciones, mensajes, recibir imágenes no deseadas de carácter sexual, risas, empujones, golpes, pellizcos, roces y apretones, intimidación y amenazas, acoso sexual (Buquet et al. 2014; Mingo y Moreno, 2015). De manera global, estos estudios estiman 
que la magnitud del sexismo en diferentes contextos universitarios de Europa y América Latina se sitúa en torno al 40\% para las mujeres y en menor medida para los hombres.

En Chile, en los últimos 5 años se ha iniciado la elaboración de diagnósticos sobre relaciones de género en la universidad que dan cuenta también de distintas expresiones de violencia en los planteles de educación superior (Santos y Kiss, 2019) y del incremento de las denuncias de acoso sexual y discriminación de género. Si bien se dispone de datos globales y estandarizados, al menos un $25 \%$ de las estudiantes universitarias en el país ha sufrido algún tipo de hostigamiento sexual, incrementándose a más de un 30\% las que han sido víctimas de maltrato sexista (Universidad de Chile, 2014; Universidad de Los Lagos, 2019; Universidad de Valparaíso, 2018). De los antecedentes recopilados respecto de los estudios sobre violencia en las universidades, se constatan al menos dos hechos: primero, en las manifestaciones de violencia al interior de la universidad destaca la dimensión de género, con una alta frecuencia para las mujeres y, además, es una violencia cruzada con otros factores de discriminación como la clase social, identidad sexual, etnia (Cho, Crenshaw y McCall, 2013); segundo, que la violencia psicológica y el acoso sexual son las dos principales manifestaciones de maltrato al interior de los recintos universitarios.

La mayoría de las universidades chilenas disponen de protocolos para atender situaciones de violencia, así lo confirma que las 30 universidades que conforman el Consejo de Rectores de las Universidades Chilenas (CRUCH) hayan implementado estas medidas. Si bien estos mecanismos son necesarios, hasta el momento se muestran como insuficientes para atender las distintas expresiones de discriminación y desigualdad sexista (Santos y Kiss, 2019) por lo que se requiere una mayor profundización en los diagnósticos de violencia que permitan incorporar medidas de prevención por áreas de conocimiento. Estos análisis específicos además permitirán adaptar los procesos curriculares y formativos incorporando la transversalidad de género con el fin de incidir en un cambio social en favor de la igualdad.

Afrontar las problemáticas de las mujeres en las universidades es complejo, en este sentido Buquet et al. (2014) lo reafirman al plantear que "el reclamo de un territorio exclusivo para la producción del conocimiento que no se vea entorpecido por el escándalo de la vida cotidiana es uno de los subtextos en la reproducción de los mecanismos de exclusión que garantizan la supremacía masculina” (p. 105). El sexismo se manifiesta en las universidades como la forma más extendida de violencia de género normalizada, siendo reiterado, sistemático y sutil. El sexismo forma parte de las relaciones sociales de la modernidad, se encuentra en el sistema patriarcal, se estructura a partir del discurso racionalista y se acoge a la noción de naturaleza para producir el mismo efecto: legitimar en las prácticas sociales la condición de sujeción y subordinación de las mujeres (Mingo y Moreno, 2015). El sexismo como expresión de la violencia de género resulta incómodo para las instituciones de educación superior, propiciando su negación inicial.

Las instituciones inmersas en la cultura de sexo/género parecen tolerar cierto tipo de conductas en la medida que su carácter sexista y discriminatorio no aparezca de manera explícita, sino vaga y opaca. Se ha caracterizado y definido al sexismo como un tipo de violencia simbólica, como una manera de relacionarse frente a otro/a. Se actualiza en cadenas de rituales de interacción en contextos socioculturales. Abarca un conjunto de comportamientos aparentemente inocuos, aceptados y como parte del juego social establecido, como la imposición de ciertos modelos de interacción social (Mingo y Moreno, 2015, 2017) en las prácticas cotidianas de relación entre hombres y mujeres, aunque 
también entre hombres con otros hombres, y entre mujeres con otras mujeres, como un sistema de significación relativamente codificado.

El espacio universitario es lugar de tensión y conflicto de género. Esta compleja situación se asocia a aspectos territoriales, áreas de conocimiento, diversidad sexual, condiciones académicas y laborales, entre otras. En las instituciones de educación superior se ponen en evidencia asimetrías de género (Buquet et al., 2014; Martín, 2017; Ross y Gatta, 2007; Santos y Kiss, 2019) encontrando el comportamiento sexista un contexto favorable tanto en la estructura jerárquica de las instituciones como en las relaciones sociales. Es por ello que se hace necesario observar y reflexionar sobre la realidad de la violencia de género en cuanto son acciones que menoscaban y restan oportunidades de desarrollo particularmente a las mujeres. Observar y analizar las situaciones de violencia en la universidad contribuye a reflexionar y establecer medidas que posibiliten un cambio social para garantizar el principio de no discriminación en los espacios universitarios.

\section{Método: Abordando la violencia de género en los espacios universitarios}

La investigación realizada responde a un estudio de caso, exploratorio y descriptivo, de carácter cuantitativo, a partir de la realización de una encuesta dirigida a los/as estudiantes de Trabajo Social de una universidad estatal, Universidad Arturo Prat (UNAP), en el norte de Chile, en Iquique. Su objetivo es detectar y analizar las distintas expresiones y contextos de violencia que pueden estar afectando a los y las estudiantes de la carrera de Trabajo Social.

Los datos para el análisis sobre violencia hacia los y las estudiantes se han obtenido de la realización de una encuesta representativa. Para ello se diseñó un cuestionario con 40 preguntas con 8 campos de interés, que van desde la identificación de los sujetos, sus características sociales y económicas, percepción de su proceso formativo, opiniones y valores sociales; además del reconocimiento de la violencia desde su identificación y experiencia, las situaciones y manifestaciones de cualquier tipo de violencia en su proceso de formación académica, las reacciones a las mismas y las acciones que consideran necesarias para atender la violencia en la universidad.

Para recabar información específica sobre las expresiones de maltrato en los procesos formativos se ha aplicado una tipología que considera tres tipos de violencia: primero, la violencia psicológica, la que comporta desde insultos y garabatos, rechazo y aislamiento, críticas referidas al trabajo, al aspecto físico y a la identidad sexual, así como intimidación y amenazas; segundo, la violencia física que incluye empujones, lanzamiento de objetos y golpes; y en tercer lugar, la violencia sexual, que atiende el hostigamiento a través de recibir mensajes o imágenes de naturaleza sexual, acoso sexual, además de situaciones donde han se han sentido obligados o han intentado forzarles para para mantener relaciones sexuales.

El universo de estudio está conformado por estudiantes de la carrera de Trabajo Social, matriculados en el segundo semestre de 2018, desagregado según nivel de estudios y sexo. Sobre esta base se elaboró una muestra representativa estratificada y aleatoria por nivel. El cuestionario fue tomado de manera simultánea en las salas de clases por nivel, elegidos/as al azar y en papel, en los meses de octubre y noviembre de 2018. La muestra se calculó para un universo de 129 estudiantes, con un nivel de confianza (z) estimado de 
un $99 \%$ y un margen de error (e) de $+/-10 \%$, obtenido un tamaño muestral de 73 estudiantes.

Cuadro 1. Distribución de la muestra

\begin{tabular}{lccc}
\hline \multicolumn{1}{c}{ NIVEL } & HOMBRES & MUJERES & TOTAL \\
\hline Primero & 5 & 14 & 19 \\
Segundo & 6 & 12 & 18 \\
Tercero & 4 & 12 & 16 \\
Cuarto & 1 & 6 & 7 \\
Quinto & 1 & 12 & 13 \\
\hline Total & 17 & 56 & 73 \\
\hline
\end{tabular}

Fuente: Elaboración propia.

\section{Resultados}

La presentación de los resultados del estudio se expone a partir de una contextualización que define el perfil de los y las estudiantes de Trabajo Social. A continuación, se han tomado tres dimensiones de análisis: primero, observar el continuo de la violencia como elemento presente en la experiencia de vida de los y las estudiantes. Segundo, la presencia de la violencia de género en los espacios formativos y, por último, el sexismo y la violencia en la universidad.

\subsection{Estudiantes de trabajo social en universidad pública del norte de Chile}

$\mathrm{El}$ acceso de las mujeres a la universidad ha sido uno de los cambios más importantes en la última década en la educación. En Chile se observa un aumento sostenido de la participación de las mujeres en la ampliación de sus estudios hasta alcanzar el 55\% de la matrícula universitaria, según datos del Departamento de Evaluación, Medición y Registro Educacional (DEMRE) para el año 2019². No obstante, este acceso está sesgado respecto a la distribución de estudiantes por área de conocimiento, observándose como aún se mantienen áreas masculinizadas y áreas altamente feminizadas, entre las que encontramos la carrera de Trabajo Social.

En la Universidad Arturo Prat, la carrera de Trabajo Social es altamente vocacional y presenta una matrícula altamente feminizada. Son estudiantes principalmente de la Región de Tarapacá, observando tres peculiaridades: un componente cultural-étnico destacado (25\% declaran pertenecer a pueblos originarios); segundo, alrededor del $21 \%$ adscribe a la diversidad sexual (población LGTBI); y tercero, algo más del 35\% manifiesta pertenecer a familias con un nivel socioeconómico bajo y el resto a un nivel medio-bajo, por lo que más de la mitad de los/as estudiantes han ingresado a la universidad acogidos a la gratuidad, condición que beneficia en mayor medida a las mujeres, puesto que 7 de cada 10 estudian bajo esta modalidad.

El estudiantado de Trabajo Social percibe que la discriminación de género en el país afecta particularmente a mujeres y personas que adhieren a la diversidad sexual. Además, comparten de manera inequívoca que el espacio universitario no es un lugar donde se promueva y garantice la igualdad, así 7 de cada 10 estudiantes de Trabajo Social sostienen

${ }^{2}$ Se puede consultar los datos completos y desagregados en

https://demre.cl/publicaciones/2020/2020-20-03-05-instrucciones-matricula 
que la universidad es un espacio donde la discriminación de género está presente, observación que comparten tanto hombres como mujeres.

\subsection{El continuo de la violencia como experiencia de vida}

La violencia de género es un problema público que afecta a la convivencia social y al desarrollo de las personas. Esta violencia ha formado parte de los procesos de socialización y se manifiesta de distintas formas a lo largo del ciclo vital de las personas y en los distintos espacios sociales, públicos y privados, con diferentes expresiones. Se observa claramente como distintas manifestaciones de violencia han estado presentes en la experiencia de vida de los y las estudiantes con rasgos claramente diferenciadores tanto por intensidad, tipos de agresión y sexo. Este hecho supone que en los procesos de formación y socialización han estado regidos por parámetros insertos en la tensión sexo/género ante las diferencias que se observan. Se aprecia (figura 1) como 7 de cada 10 estudiantes declaran haber sufrido algún tipo de maltrato a lo largo de su vida mostrándose una distribución diferenciada para hombres y mujeres: la violencia ha afectado en mayor medida al proceso vital de las mujeres y se vincula con mayor intensidad al hostigamiento sexual.

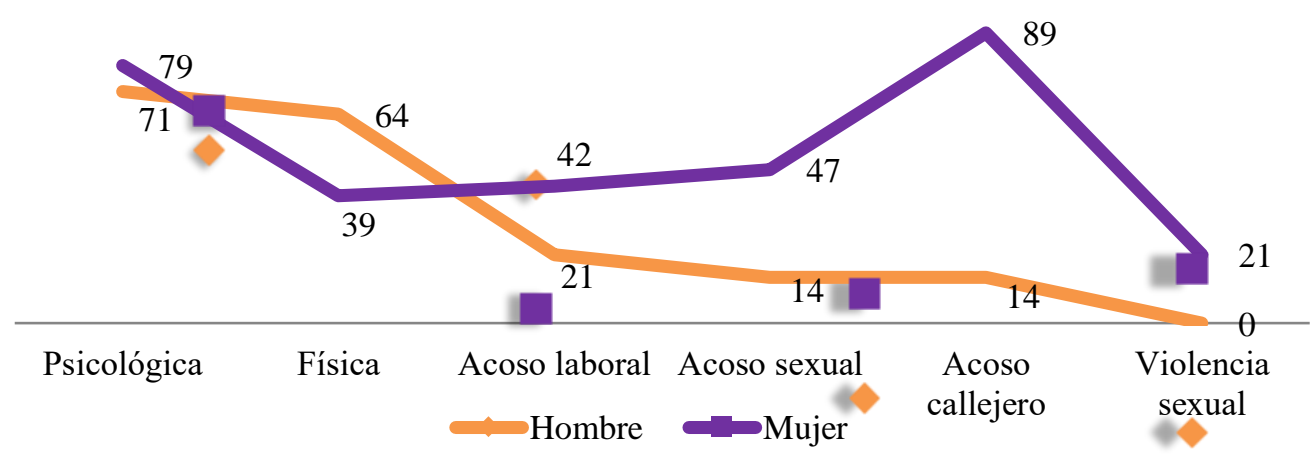

Figura 1. Resultados de tipos de violencia que han afectado a estudiantes de Trabajo

Social (en porcentaje)

Fuente: Elaboración propia.

De acuerdo a la figura 1, la violencia psicológica es el tipo de agresión con mayor incidencia, sin que se observen diferencias por sexo. En cambio, en el resto de los tipos de violencia se aprecian brechas significativas para hombres y mujeres. El acoso laboral es más frecuente entre las jóvenes ( $42 \%$ mujeres y $21 \%$ hombres), brecha de género que aumenta cuando el tipo de violencia tiene connotación de índole sexual y donde las mujeres alcanzan cifras preocupantes. En efecto, los resultados arrojan que 1 de cada 2 estudiantes declara haber sido víctima de acoso sexual, en tanto que esta situación la han vivenciado el $14 \%$ de los hombres. El acoso callejero es el que tiene mayor magnitud afectando al $89 \%$ de las mujeres; y la violencia sexual, si bien tiene una ocurrencia de un $15 \%$, cuando se desagrega por sexo, se observa que afecta al $21 \%$ de las estudiantes.

Estas cifras revelan como la cultura patriarcal y los estereotipos de género están fuertemente arraigados en el proceso vital de los/as estudiantes al observar una mayor incidencia de la violencia a lo largo de la vida de las mujeres lo que las va posicionando socialmente. La normalización de las conductas violentas y los comportamientos de dominación en la socialización de los hombres también se hace presente. Estos datos permiten reconocer la normalización de la violencia en las relaciones sociales. También en 
clave de construcción de identidades masculinas se observa la violencia física, exhibición de fuerza más extendida entre los hombres.

En cuanto a los espacios de convivencia e interacción social que identifican los/as estudiantes como lugares donde han vivido situaciones de violencia son muy variados y permiten afirmar que cualquier lugar propicia la discriminación y el maltrato, aunque los que se identifican con mayor incidencia y riesgo son la calle $(67 \%)$ y los transportes públicos (64\%). Este hecho se relaciona por un lado con la cultura sexista y por otro con la impunidad, aspectos que se imponen en estas relaciones esporádicas y transitorias en espacios públicos, donde la mujer es más vulnerable.

Los espacios íntimos y afectivos donde se insertan las relaciones familiares donde se deberían cumplir funciones fundamentales de socialización, afecto y protección, ofrecen un espacio privado de riesgo y una educación sexista. Es elevado el maltrato al interior del hogar, puesto que la mitad de los/as estudiantes declara haber sufrido esta situación, siendo bastante más frecuente para la población femenina.

Los lugares de interacción social y vida cotidiana como son los entornos de diversión y los espacios laborales se pueden considerar de alto riesgo, ya que 1 de cada 3 estudiantes declara haber sufrido maltrato en alguno de ellos, situación que también evidencia mayor gravedad para las mujeres. Los espacios educativos y la universidad no están libres de violencia, en este caso, el 29\% de las y los estudiantes de la universidad manifestaron haber sufrido algún tipo de agresión en estos contextos, pero se distinguen algunos matices. Las mujeres acusan estar expuestas a situaciones de violencia en todos los ámbitos del quehacer universitario; mientras que los hombres parecen estar más expuestos a las agresiones en la sala de clases y en los grupos de trabajo entre estudiantes (figura 2).

No hay un comportamiento claro por parte de las/os estudiantes para responder a las situaciones de violencia. Las mujeres presentan una mayor tendencia a silenciarla, así el $39 \%$ de las estudiantes que han declarado ser víctimas manifiestan no haber hecho pública esta situación, frente al silencio del 14\% de los estudiantes en la misma situación. Entre los argumentos que se relacionan con esta conducta están el no saber qué hacer, sentir vergüenza y sentir miedo. Contrariamente, quienes sí reaccionaron frente a estos sucesos, indicaron que lo comunicaron a familiares (26\%), a sus amistades $(29 \%)$ o solicitaron ayuda psicológica (24\%), siendo la mujer la que más se inclina por estas opciones. Muy pocos buscaron apoyo legal (5\%) o interpusieron una denuncia en la policía (11\%). Estas cifras reflejan como las experiencias de violencia son frecuentes, regulares, se tienden a silenciar y entran dentro de relaciones sociales normalizadas. La falta de acciones preventivas, de información y de protocolos dejan a la víctima sin posibilidades de denunciar estos ataques. De esta forma se percibe como se da una respuesta privada a un problema social. La violencia se oculta bajo el silencio y especialmente este mutismo dificulta su prevención y atención. 


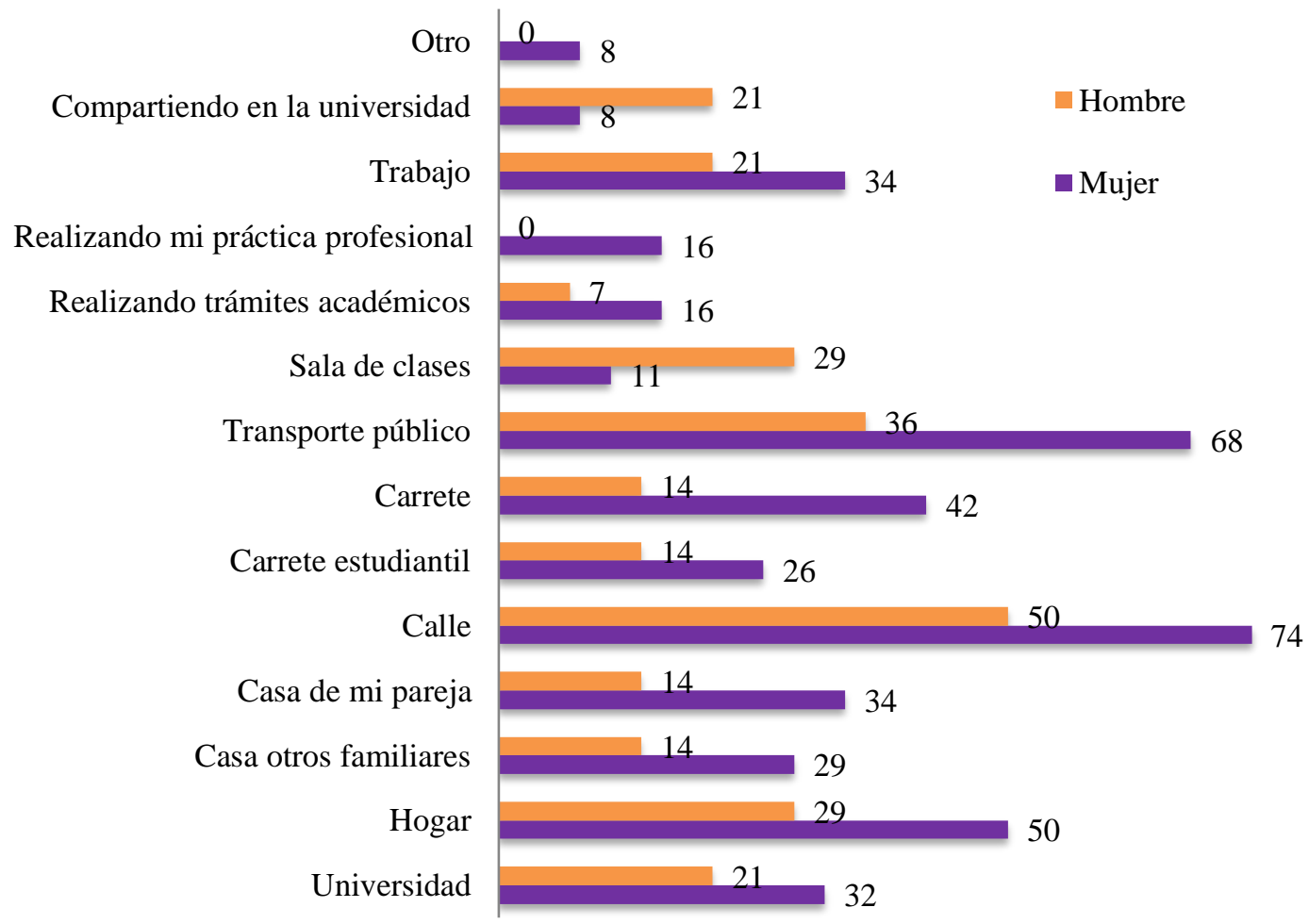

Figura 2. Resultados de lugares donde los y las estudiantes han sido víctimas de violencia (en porcentaje) Fuente: Elaboración propia.

\subsection{Violencia de género en los procesos formativos}

La educación sexista y la violencia de género como mecanismo de comunicación, interacción y aprendizaje social ha sido un continuo en la vida de los y las estudiantes. Las niñas y las jóvenes presentan mayor riesgo y vulnerabilidad en sus procesos de desarrollo, en este sentido las agencias de socialización, como la familia y el colegio, lugares fundamentales en los procesos de formación como personas y sujetos sociales, están mediadas por valores culturales donde prevalece una cultura patriarcal. Es por ello que se ha considerado importante indagar en la experiencia de los distintos tramos formativos, desde la educación básica, media y universitaria. Los datos obtenidos permiten afirmar que los espacios de formación y socialización de los jóvenes estudiantes han sido lugares donde los aprendizajes sociales están relacionados con situaciones intimidatorias y agresivas que insertan a los sujetos sociales en su cultura, encontrando el sexismo normalizado aun cuando se aprecian significativas diferencias en cuanto a tipos de agresión y ciclo formativo.

Ingresar en la educación básica constituye un hito relevante en el desarrollo integral de los seres humanos al ser un espacio de socialización secundaria que abre un nuevo espectro de relaciones humanas. Ante la consulta que sitúa a las y los estudiantes en la exposición a violencia durante esta etapa de su vida, estos declaran haberla vivido en distintas situaciones en el colegio, alcanzando la violencia psicológica al 70\% de los niños y niñas (figura 3). Esta alta frecuencia presenta diferentes tendencias según sexo. La violencia psicológica está muy extendida, especialmente aquella ligada a formas de comunicación 
verbal como son el insulto, garabato y gritos, la que es vivenciada en mayor medida por los niños. En cambio, las críticas, tanto por expresar opinión como por apariencia física, así como el rechazo y aislamiento, formas más sutiles y soterradas de agresión, afectan en mayor medida a las niñas (disciplinamiento). La violencia física se asocia en mayor medida con la formación y comportamiento de los varones. Y el hostigamiento sexual se muestra más en la experiencia de las niñas (16\%). La violencia sexual, en torno al $6 \%$, no presenta diferencias significativas por género, afectando por igual a niños y niñas.

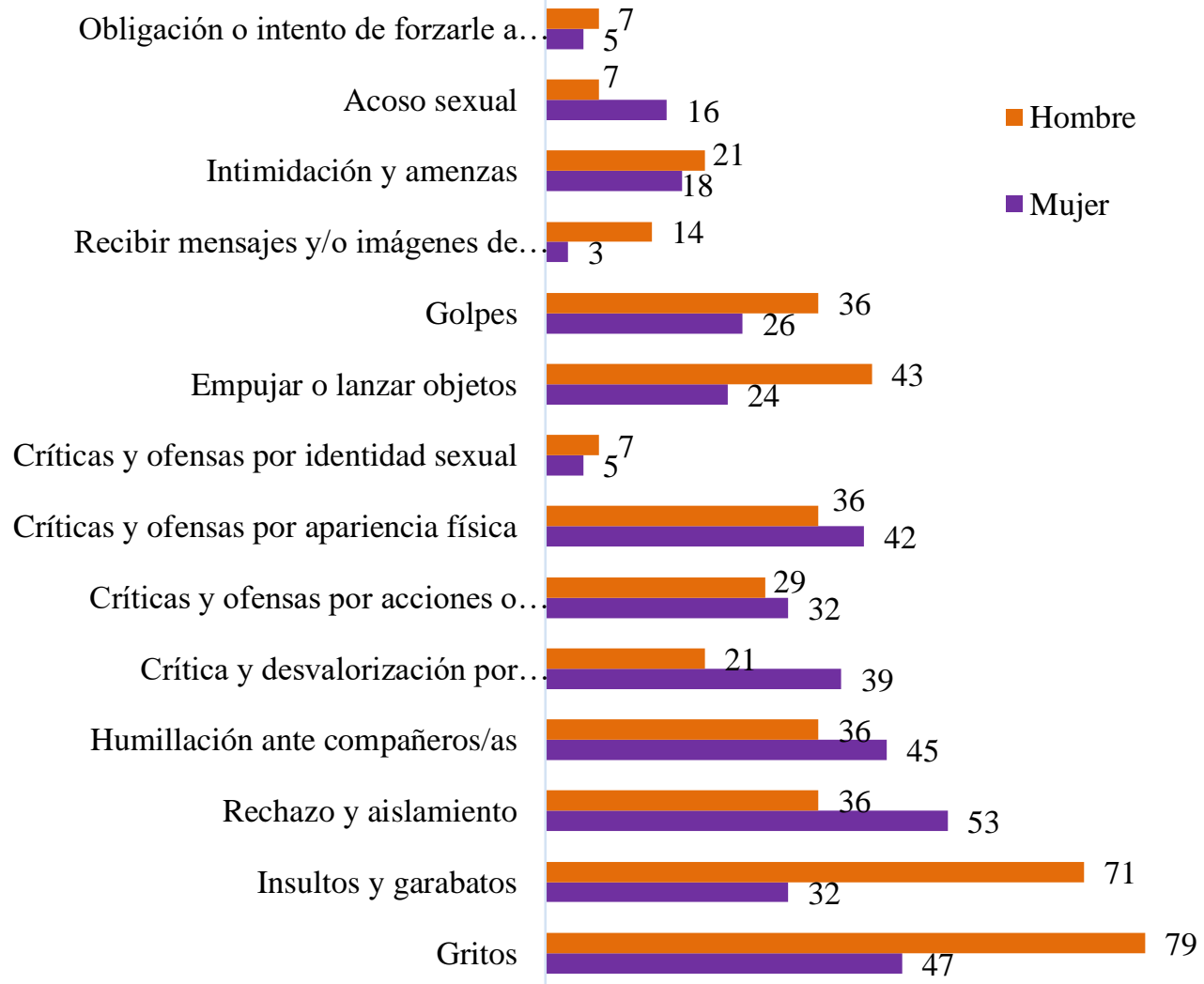

Figura 3. Resultados de violencia durante la Educación Básica (en porcentaje)

Fuente: Elaboración propia.

A lo largo de la educación media, comparativamente con el ciclo escolar anterior, se registra una menor frecuencia en las agresiones que declaran los y las estudiantes al alcanzar las experiencias de violencia alrededor del 50\% de los/as encuestados/as (figura 4). Entre los 14 y 18 años, los hombres declaran en mayor medida estar expuestos a violencia tanto física como psicológica. En este grupo etario y formativo, se destaca como las mujeres presentan mayor nivel de violencia psicológica del tipo rechazo y críticas por apariencias que los hombres, mientras que son los jóvenes los que revelan sufrir mayor hostigamiento sexual respecto de mensajes e imágenes de esta índole (43\%). No se observan diferencias de género respecto al acoso sexual, con una incidencia de 1 de cada 4 estudiantes, así como tampoco se observan diferencias respecto a la violencia sexual que se mantiene en torno al $6 \%$. 


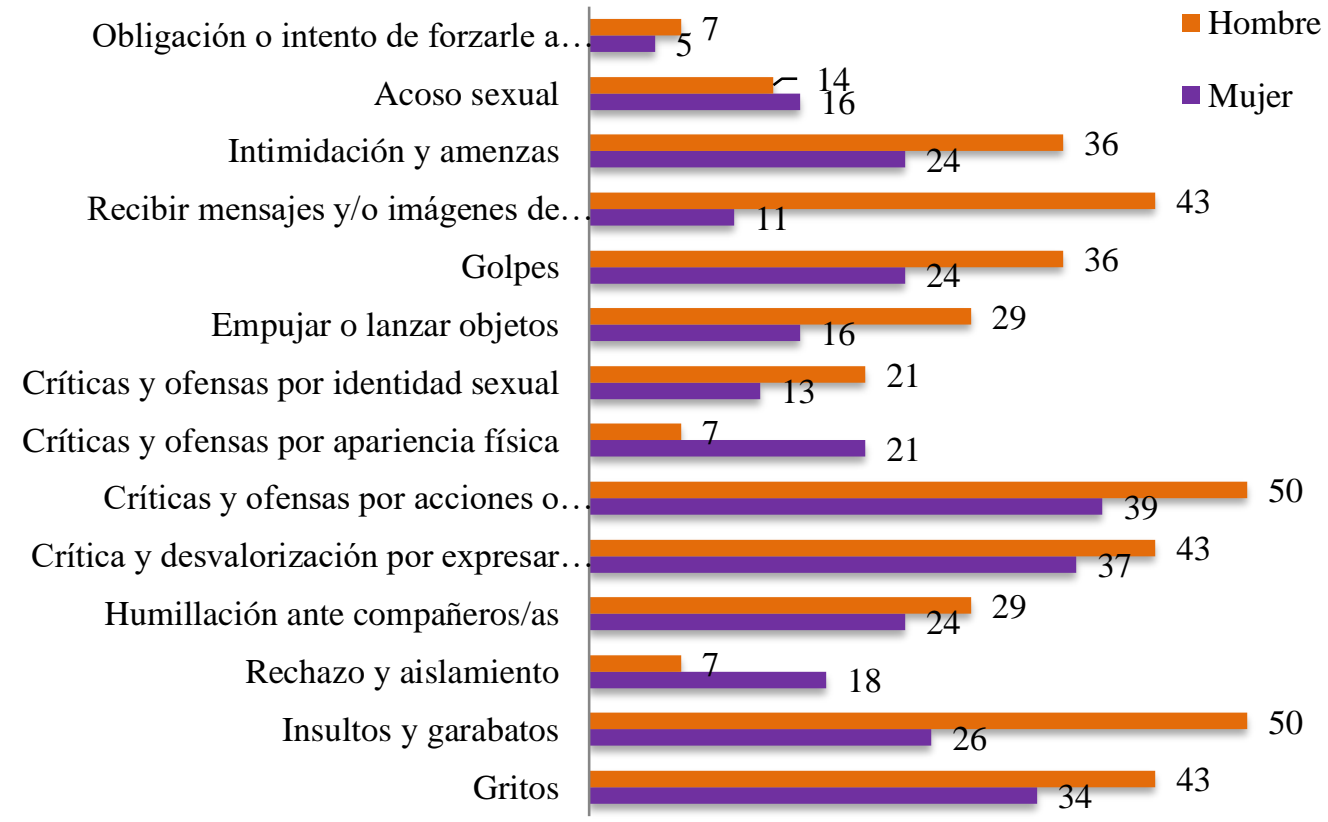

Figura 4. Resultados de violencia durante la Educación Media (en porcentaje)

Fuente: Elaboración propia.

La violencia observada a lo largo de los primeros ciclos de educación ha estado muy presente en la vida de los y las estudiantes y muestra diferencias por sexo. Los estereotipos de género y la violencia están se manifiestan en los procesos de aprendizaje y de socialización de los y las jóvenes. La violencia que declaran los jóvenes tiende a ser en mayor medida física y psicológica, siendo esta última la que presenta mayores sutilizas para las mujeres, al ser más opaca y silenciosa, en torno a las críticas por opiniones y por su apariencia física. En cuanto a la violencia sexual, se registran diferencias significativas por género, el hostigamiento sexual ha estado muy presente en educación básica para las estudiantes (niñas) y en educación media afecta por igual a hombres y mujeres (15\%).

\subsection{Sexismo y violencia en la universidad. La experiencia de los y las estudiantes de Trabajo Social}

A la universidad ingresan los y las estudiantes de Trabajo Social conocedores ya de pautas culturales y de comunicación configuradas desde patrones sexistas y situadas en una lógica operativa que define las relaciones sociales jerárquicas basadas en las estructuras de poder (verticales) y también en la subordinación femenina (horizontales, en la relación de pares hombre/mujer). Para cuando ingresan a la universidad, ya están dados los elementos para operar desde el denominado sexismo performativo (Mingo y Moreno, 2015) donde interactúan públicamente los y las jóvenes desde los aprendizajes sociales. Es la puesta en escena social de la configuración de las relaciones basadas en la tensión sexo/género.

Como se ha señalado anteriormente, la universidad no es un espacio neutral al margen de las estructuras sociales y políticas de la sociedad. Es un espacio de formación y convivencia social, con una marcada estructura institucional y jerárquica, donde las brechas de género afectan al conjunto de la comunidad universitaria (Santos y Kiss, 2019). 
En la universidad, los y las estudiantes de Trabajo Social viven distintas expresiones de violencia. Los datos recabados señalan que, si bien la magnitud de la violencia disminuye, respecto a las etapas formativas anteriores, en este momento se evidencia una clara expresión y despliegue de la violencia de género, estando las estudiantes de Trabajo Social más expuestas que sus compañeros de estudio. La violencia psicológica afecta a casi un tercio del estudiantado de Trabajo Social y asciende a un $40 \%$ en las mujeres. La violencia física, con baja incidencia, es declarada por el 7\% de los estudiantes; y la violencia sexual incide en el 3\% de las estudiantes (figura 5).

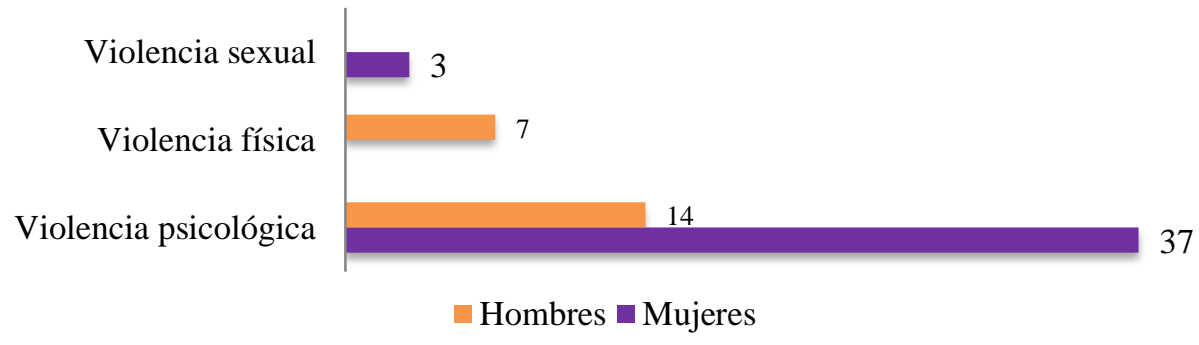

Figura 5. Resultados de violencia en la Universidad (en porcentaje) Fuente: Elaboración propia.

Respecto a quienes son las personas que agreden a los y las estudiantes, los datos son muy relevantes al evidenciar que si bien entre los diferentes estamentos universitarios (académico, funcionario y estudiantil) se ejerce violencia hacia los y las estudiantes, esta tiende a concentrase en las relaciones entre pares. Es la violencia psicológica y sexista la que se expresa a través de insultos, rechazo, aislamiento, críticas y desvalorización, es decir, son las relaciones interpersonales las que se sitúan en el eje de la violencia que se ejerce, afectando las relaciones entre estudiantes principalmente (eje horizontal).

Tal y como se muestra en la figura 6 , los dos nudos críticos de la violencia se sitúan en las relaciones entre estudiantes, y entre profesores/as - estudiantes. El primero, en la relación entre pares, es la que concentra mayor atención, puesto que es en estas interacciones donde se encuentran los mayores conflictos, básicamente relacionados con el respeto y la comunicación, hablarían de aprendizajes y pautas de conductas que alteran la convivencia y que tienen un fuerte componente cultural. Cabe destacar que en las relaciones entre estudiantes no se advierte violencia física ni sexual.

En cuanto a la relación entre académicos/as y estudiantes (eje jerárquico, vertical) se encuentra la violencia psicológica, si bien con una baja frecuencia que no pasa desapercibida, mostrándose fundamentalmente en pautas de comunicación que dañan al estudiantado al residir fundamentalmente en la crítica hacia los mismos, la que es acusada en mayor medida hacia las mujeres.

$\mathrm{El}$ acoso sexual y la violencia sexual son declarados por las mujeres (3\%), identificando a profesores y otros profesionales que participan directamente del proceso formativo de las estudiantes, lo que nos lleva a reflexionar respecto a las posiciones de poder (jerárquica y vertical) en este tipo de maltrato hacia las estudiantes. 


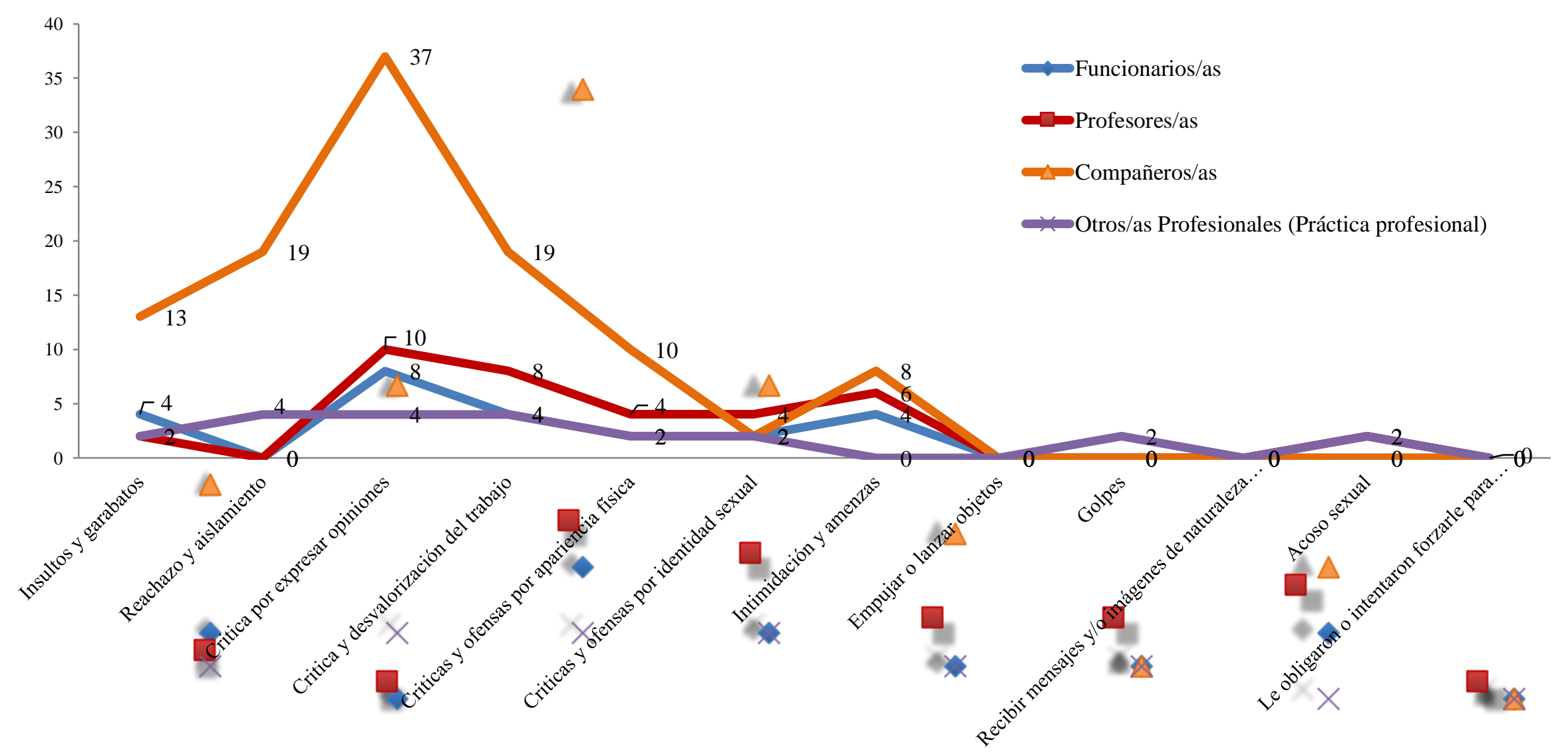

Figura 6. Resultados de violencia hacia los y las estudiantes en relación a los diferentes estamentos (en porcentaje) Fuente: Elaboración propia. 
La violencia está muy presente en las relaciones entre estudiantes (horizontalidad) afectando a mujeres y varones; mientras que la violencia contra las mujeres tiene una doble vertiente, por un lado, un eje horizontal (entre pares) y un eje vertical (jerárquico) por lo que las estudiantes de Trabajo Social están más expuestas a sufrir violencia que sus compañeros.

\section{Conclusiones: Reconocer el sexismo en la universidad y actuar para su erradicación.}

Las universidades son espacios donde la discriminación y el sexismo están presentes (Buquet et al., 2014; Martín, 2017; Santos, 2019) y enmarcados en una cultura que tiende más a reproducir la desigualdad de género que a erradicarla lo que reduce las oportunidades de desarrollo de las mujeres.

En nuestro país hay mayor interés por determinar las causas y formas en la que se expresa la violencia en las instituciones de educación superior. En este sentido, esta investigación ha permitido conocer las expresiones de este fenómeno a través de las vivencias de estudiantes de la carrera de Trabajo Social.

De acuerdo a los resultados, podemos afirmar que la violencia ha estado presente en la trayectoria de vida de las y los estudiantes y tiene su base en la estructura sexista y patriarcal de nuestra cultura. Subyace de manera permanente, opaca y normalizada; y emerge en aquellos contextos de aprendizaje e interacción social, en los distintos espacios sociales, públicos y privados, manifestándose de diversas formas y en distintos grados, según se analiza a partir de su desagregación por sexo y tramo etario y formativo. En todos los contextos de interacción social las jóvenes corren un mayor riesgo de ser víctimas de violencia que los hombres (calle, transporte, trabajo, espacios de diversión, lugares de formación y familia).

En los procesos formativos, fuera del contexto del núcleo primario, la violencia presenta una alta frecuencia. En consistencia a este planteamiento, Neut (2017) señala que en Chile la violencia entre pares se manifiesta de manera más extendida entre los estudiantes de básica que entre los de educación media y que los varones presentan mayores índices de violencia y victimización respecto del que muestran las mujeres. Por otra parte, los resultados de la IX Encuesta Nacional de Juventud (INJUV, 2019), indican que la escuela es el lugar donde más violencia física se reporta y con mayor incidencia en los estratos de bajos recursos.

Entre los principales factores que influyen en la instalación de conductas violentas podemos situar las experiencias a las que han sido expuestos los/as jóvenes en el espacio privado, íntimo y afectivo de la familia. En efecto, los niños y niñas que sufren violencia directa o indirecta en sus hogares, tienen dificultades para desarrollar habilidades sociales, establecer relaciones con sus pares y profesores, además de presentar otros trastornos a nivel físico y emocional. En este mismo sentido, Sepúlveda García (2006) aborda la transmisión transgeneracional de la violencia en tanto la familia es el agente socializador más importante. Los niños y niñas que crecen en entornos maltratantes aprenden a interiorizar estos valores, entre los que prevalecen los estereotipos de género, la desigualdad entre hombres y mujeres y la violencia en la resolución de los conflictos. 
Hemos observado que la violencia física tiende a disminuir a medida que los y las estudiantes se van incorporando a niveles superiores de educación. Situación inversa se presenta con la violencia psicológica y sexual, mostrando como las mujeres progresivamente están más expuestas al sexismo, el hostigamiento y la violencia sexual. Es decir, los patrones culturales aparecen cristalizados y se despliegan los valores y estereotipos de género aprendidos.

En la universidad, en el caso de estudiantes de Trabajo Social, la violencia está presente y con una alta incidencia de algo más del 30\% en su expresión psicológica y sexista, la que se puede interpretar desde una perspectiva de género, pues representa los aspectos esenciales de la estructura social en un marco de relación y de poder sexo/género (Fernández Moreno, Hernández Torres y Paniagua Suárez, 2005). Las asimetrías en las relaciones de poder podrían incidir en las formas y frecuencias en las que los jóvenes reconocen haber experimentado situaciones de violencia. Precisamente, la horizontalidad en las interacciones entre pares pareciera favorecer que aparezcan en alto porcentaje las agresiones de tipo físicas y psicológicas, en tanto apuntan a generar sistemas de relación jerárquicas basadas en la dominación del otro, a través de la fuerza, la intimidación y la discriminación. Por otra parte, en las relaciones adulto-niño o profesor-estudiante, la posición de sumisión está relacionada con el estatus que comúnmente adquiere el agresor (verticalidad) en una sociedad adultocentrista y androcentrista.

Si bien las situaciones que constituyen violencia sexual (3\% mujeres) son declaradas en menor frecuencia, estas no dejan de ser preocupantes, más aún cuando persisten conductas sexistas y misóginas en todos los ámbitos de participación de las estudiantes que afectan a su autoestima y desarrollo. La violencia sexual, a través del hostigamiento y acoso, está normalizada, obedeciendo a construcciones estereotipadas y situando a las mujeres como responsables/provocadoras (Aguilar Ródenas et al., 2009), hecho que incide en minimizar su importancia, tratarlo como un problema privado, subjetivo y opaco, que desanima a la denuncia ante instancias que otorgan descrédito a la situación de las víctimas, contribuyendo a la cultura cómplice del silencio (Mingo y Moreno, 2016). Hasta ahora este es el panorama habitual en las instituciones de educación superior, donde se observa una creciente conciencia respecto de situaciones que constituyen violencia de género $y$, sin embargo, no se acompañan de las medidas necesarias. Lo anterior, podría relacionarse con la sensación de impunidad y la necesidad de incorporar algunas conductas normalizadas dentro de las tipificaciones legales que constituyen acoso, discriminación y violencia.

Las medidas de prevención y atención a la violencia de género en las universidades, deben ser efectivas y pertinentes, atendiendo las particularidades de cada contexto. El espacio universitario debe valorar el pluralismo, la diversidad y el respeto, principios básicos en la formación de profesionales integrales, incorporando en su currículum la perspectiva de género, desarrollar competencias de análisis crítico e intervención y resignificar las experiencias de violencia que han afectado la construcción social de sus estudiantes. En la formación en Trabajo Social ello resulta imprescindible. Asimismo, es necesario generar análisis comparados entre distintas escuelas de Trabajo Social que posibiliten una mayor profundización en las expresiones de violencia que afectan al estudiantado de este campo de estudio y en las repercusiones como futuros profesionales. 


\section{Referencias}

Aguilar Ródenas, C., Alonso Olea, M. J., Melgar Alcatud, P., y Molina Roldán, S. (2009). Violencia de género en el ámbito universitario: medidas para su superación. Pedagogía Social. Revista Interuniversitaria, 16, 85-94. https://doi.org/10.7179/PSRI_2009.16.06

Arendt, H. (2018). Sobre la violencia. Alianza Editorial.

Arruza, C (2017). Reflexiones degeneradas. Sobre patriarcado y capitalismo. En C. Arruza (Ed.), Género y capitalismo. Debates en torno a reflexiones degeneradas (pp. 23-54). Grupo de Estudios Feministas.

Bosch-Fiol, E. y Ferrer-Pérez, V. (2012). Nuevo mapa de los mitos sobre la violencia de género en el siglo XXI. Psicothema, 24(4), 548-554

Buquet, A. (2011). Transversalización de la perspectiva de género en la educación superior. Problemas conceptuales y prácticos. Perfiles Educativos, 33, 211-225.

Buquet, A., Cooper J. A., Mingo, A. y Moreno H. (2014). Intrusas en la universidad. Universidad Nacional Autónoma de México.

Carballeda, A. (2017). Escenarios sociales, intervención social y acontecimiento. Universidad Nacional de Moreno Editora.

Castells, M. y Subirats, M. (2010). Hombres y mujeres ¿̇Un amor imposible? Alianza Editorial.

Cho, S., Crenshaw, K. y McCall, L. (2013). Toward a field of intersectionality studies: Theory, applications, and praxis. Signs. Journal of Women in Culture and Society, 38(4), 785-810. https://doi.org/10.1086/669608

Cobo, R. (2019). Hacia una nueva política sexual. Las mujeres ante la relación patriarcal. Editorial Planeta.

Connell, R. W. y Messerschmidt, J. W. (2005). Hegemonic masculinity: Rethinking the concept. Gender \& Society, 19(6), 829-859. https://doi.org/10.1177/0891243205278639

De Miguel, A. (2015). Neoliberalismo sexual. El mito de la libre elección. Cátedra.

Fernández Moreno, S. Y., Hernández Torres, G. E. y Paniagua Suárez, R. E. (2005). Violencia de género en la Universidad de Antioquia. Universidad de Antioquia.

Instituto Nacional de la Juventud. (2019). Novena encuesta nacional de juventud. INJUV.

Martin, S. (2018). Querer y poder: (Des)Igualdad en la universidad pública española. Contextos Educativos. Revista de Educación, 21, 11-34. http://doi.org/10.18172/con.3304

Mingo, A. y Moreno, H. (2015). El ocioso intento de tapar el sol con un dedo: Violencia de género en la universidad. Perfiles Educativos, 37(148), 138-155.

https://doi.org/10.22201/iisue.24486167e.2015.148.49318

Mingo, A. y Moreno, H. (2017). Sexismo en la universidad. Estudios Sociológicos, 35(105), 571-595. https://doi.org/10.24201/es.2017v35n105.1434

Neut, P. (2017). Las violencias escolares en el escenario educativo chileno. Análisis crítico del estado del arte. Calidad en la Educación, 46, 222-247.

https://doi.org/10.4067/So7 18-45652017000100222.

Osborne, R. (2009). Apuntes sobre violencia de género. Bellaterra.

Ross, P. y Gatta, M (2007). Gender (in)equity in the academy. Subtle mechanisms and reproduction of inequality. Research in Social Stratification and Mobility, 27(3), 177-200.

https://doi: 10.1016/j.rssm.2009.04.005 
Santos, A. (2019). ¿La igualdad de género en la Universidad para cuándo? Movimiento feminista universitario en Chile. Revista Iberoamericana. América Latina-España-Portugal, 72, 223-245.

Santos, A. y Kiss, D. (2019). Brechas de género, demandas feministas y políticas con igualdad. Reflexiones en torno a la experiencia chilena. Universidad Nacional de Rosario.

Segato, R. (2016). La guerra contra las mujeres. Traficante de Sueños.

Sepúlveda García de la Torre, A. (2006). La violencia de género como causa de maltrato infantil. Cuadernos de Medicina Forense, 44, 149-164. https://doi.org/10.4321/S1135-76062006000100011

Troncoso, L., Follegati, L. y Stutzin, V. (2019). Más allá de una educación no sexista: Aportes de pedagogías feministas interseccionales. Pensamiento Educativo. Revista de Investigación Educacional Latinoamericana, 56(1), 1-5. https://doi.org/10.7764/PEL.56.1.2019.1

Universidad de Chile. (2014). Del biombo a la cátedra. Universidad de Chile.

Universidad de Los Lagos. (2019). Diagnóstico de género de la Universidad de Los Lagos. Universidad de Los Lagos.

Universidad de Valparaíso. (2018). Análisis sobre las relaciones de género en la Universidad de Valparaíso. Universidad de Valparaíso.

Velázquez, S. (2006). Violencias cotidianas, violencias de género. Escuchar, comprender, ayudar. Paidós.

Vivero, L. (2017). Aportes del pensamiento latinoamericano al trabajo social. Revista Sophia Austral, 20, 71-81. https://doi.org/10.4067/S0719-56052017000200071

\section{Breve CV de las autoras}

\section{Carolina Garcés Estrada}

Trabajadora Social, Universidad de Concepción. Magister en Trabajo Social y Políticas Sociales por la Universidad de Concepción, Master en Metodologías de la Investigación en Ciencias Sociales y Magister en Evaluación de Programas y Políticas Públicas por la Universidad Complutense de Madrid. Se desempeñó como académica de la Universidad de Magallanes entre 2001 y 2015, donde fue Jefa de Carrera de Trabajo Social, Directora del Departamento de Ciencias Sociales y Editora General de la Revista Sophia Austral. Actualmente es académica e investigadora de la Facultad de Ciencias Jurídicas y Políticas de la Universidad Arturo Prat. Sus áreas de interés e investigación comenzaron con temas de vejez y envejecimiento; relaciones intergeneracionales, actualmente se ha enfocado en temas de feminismo, género y cuidados. ORCID ID: https://orcid.org/0000-0003-38543767. Email:cgarces@unap.cl

\section{Antonia Santos Pérez}

Socióloga por la Universidad Complutense de Madrid. Doctora en Ciencia Política, Universidad de Salamanca (España). Ha ocupado cargos de Jefa de Carrera de Sociología, Directora del Departamento de Ciencias Sociales, Directora de la Unidad de Estudios de Opinión Pública, Decana de la Facultad de Ciencias Jurídicas y Políticas, Directora de la Dirección de Equi dad de Género y Diversidad, de la Universidad Arturo Prat. Académica e investigadora de la Facultad de Ciencias Jurídicas y Políticas. Sus líneas de interés son representación política, género, políticas públicas con igualdad. Actualmente Coordina la Comisión de Igualdad de Género del CRUCH realizando diversas investigaciones sobre 
brechas de género y políticas de igualdad en las universidades chilenas. ORCID ID: https://orcid.org/0000-0001-7352-6941. Email: asantos@unap.cl

\section{Loreto Alejandra Castillo Collado}

Trabajadora Social, Universidad de Valparaíso. Magíster en Ciencias Sociales con mención en Políticas Sociales por la Universidad Arturo Prat. Además de especialización en Diplomado en Intervención Familiar, Diplomado en Planificación Estratégica y Gestión Universitaria, Diplomado en Planificación y Gestión Curricular y Pos título en Mediación Social de Conflictos. Académica e investigadora, Facultad de Ciencias Jurídicas y Políticas. Ha integrado el Núcleo de Estudios Criminológicos de la Frontera. Ha sido Directora de la Carrera de Trabajo Social, Directora de Calidad y Directora de Docencia de la Facultad de Ciencias Jurídicas y Políticas. Se ha desempeñado como Directora General de Vinculación con el Medio y Relaciones Institucionales de la UNAP. ORCID ID: https://orcid.org/0000-0003-3403-4164. Email: lorcasti@unap.cl 